\title{
Lumen
}

Selected Proceedings from the Canadian Society for Eighteenth-Century Studies

\section{Taverne sous surveillance : conditions d'émergence de nouveaux espaces de divertissement semi-publics au Québec (1764-1825)}

\section{Mathieu Perron}

Volume 37, 2018

URI : https://id.erudit.org/iderudit/1042232ar

DOI : https://doi.org/10.7202/1042232ar

Aller au sommaire du numéro

Éditeur(s)

Canadian Society for Eighteenth-Century Studies / Société canadienne d'étude du dix-huitième siècle

ISSN

1209-3696 (imprimé)

1927-8284 (numérique)

Découvrir la revue

Citer cet article

Perron, M. (2018). Taverne sous surveillance : conditions d'émergence de nouveaux espaces de divertissement semi-publics au Québec (1764-1825). Lumen, 37, 215-230. https://doi.org/10.7202/1042232ar 


\title{
Taverne sous surveillance : conditions d'émergence de nouveaux espaces de divertissement semi-publics au Québec $(1764-1825)^{1}$
}

\author{
Mathieu Perron \\ Université du Québec à Trois-Rivières
}

Micheal Eamon a bien souligné le rôle du coffeehouse et de l'imprimé dans la construction d'une identité loyaliste britannique nordaméricaine à Québec et à Halifax dans les années 1780-1790². Les observations de Eamon concernant les coffeehouses demeurent toutefois parcellaires et invitent à approfondir l'étude. Pour sa part, Julia H. Roberts a pris pour cadre le Haut-Canada, une société angloaméricaine arrachée à la forêt entre 1790 et 1840 . Les tavernes des pionniers hauts canadiens abriteraient une vie sociale marquée par l'autorégulation ainsi qu'une mixité négociée de genre, de statut social et d'ethnie négociées. On retrouverait dans ces bois, pour l'essentiel, le type de mixité sociale qui a été souligné par Benjamin L. Carps pour New York durant la période prérévolutionnaire ${ }^{4}$ Là où en ville

1. L'auteur tient à remercier les évaluateurs anonymes, le comité de rédaction de Lumen ainsi qu'Alexandre T. Lamarche pour leurs commentaires. Par ailleurs, cette contribution puise dans certains de nos travaux antérieurs, notamment Mathieu Perron, Le "Parlement du peuple» : enjeux politiques et sociaux des tavernes, auberges et coffeehouses du district de Québec (1759-1775), mémoire de maîtrise, Sherbrooke, Université de Sherbrooke, 2014.

2. Michael Eamon, Imprinting Britain, Newspapers, Sociability, and the Shaping of British North America, Montréal/Kingston, McGill/Queen's University Press, 2015.

3. Julia Roberts, In Mixed Company: Taverns and Public Life in Upper Canada, Vancouver, University of British Columbia Press, 2009, p. 5, 8-10, 101-119 et 138-162.

4. Benjamin L. Carp, Rebels Rising: Cities and the American Revolution, Oxford, Oxford University Press, 2007, p. 53 et 63. 
cette forme de sociabilité aurait présidé à une mobilisation politique insurrectionnelle, au Haut-Canada elle aurait participé à l'établissement d'une société pionnière mixte et harmonieuse. Comme le relève Mary Anne Poutanen pour Montréal, les formes d'espaces de divertissements semi-publics urbains sont loin d'être univoques ${ }^{5}$. Si, d'un côté, on observe une culture élitaire, réglementée et ordonnée de la taverne, d'un autre côté on constate tout un univers d'établissement «désordonnés» (disorderly house) dont l'existence est assujettie à un jeu de négociation communautaire ${ }^{6}$. Il existe des espaces de divertissement respectables et d'autres qui le sont moins; il existe des lieux fameux (fama), mais aussi des lieux malfamés. Cette dichotomie s'appuie d'abord sur un discours de la respectabilité, mais s'alimente aussi à une anxiété sociale et politique montante.

Cette crainte de la déchéance sociale s'exprime, selon Nancy Christie, de manière aiguë au cours des années contre-révolutionnaires (1780-1820). Les autorités ont alors le projet de resserrer l'encadrement du pouvoir patriarcal sur l'espace privé familial afin de consolider l'attachement symbolique et surtout juridique des chefs de famille au pouvoir impérial. Cette stratégie constitue une tentative d'endiguement des influences de la pensée de John Locke et de l'individualisme, jugées séditieuses, que l'on croit suppurer de la république voisine? Nous verrons comment l'autorité coloniale subordonne par le truchement d'une augmentation de son pouvoir de surveillance des tenanciers en superviseur d'un espace transitoire d'échange et de mobilité ${ }^{8}$. Les deux premières sections s'appuient sur une lecture attentive et croisée d'un corpus de sources incluant gazettes, correspondances officielles et privées, récits de voyage, mémoires, licences, certificats, reconnaissances et ordonnances. Nous proposons de dégager les conditions d'émergence d'un discours officiel de la méfiance et de la surveillance face à une culture commerciale de la taverne au Québec

5. Mary Anne Poutanen, Beyond Brutal Passions. Prostitution in Early NineteenthCentury Montreal, Montréal/Kingston, McGill/Queen's University Press, 2015, p. 33-76, 107 et 182-189.

6. Nancy Christie, "He is the master of his house": Families and Political Authority in Counterrevolutionary Montreal», The William and Mary Quarterly, 70, 2,2013, p. 363 .

7. Ibid., p. 341-370 et 436 .

8. Allan Greer, Habitants et patriotes : la rébellion de 1837 dans les campagnes du Bas-Canada, Montréal, Boréal, 1997, p. 6o et 65-66. 
depuis le début du gouvernement civil en 1764 jusqu'à 1825. Cette dernière date marque une rupture dans la retranscription des verbatim de témoignages dans les notes de banc du juge en chef du district de Montréal, James Reid. Dans un premier temps, nous traiterons des conditions présidant à la constitution d'une culture de la taverne élitaire après la Conquête. Nous discuterons du rôle de ces espaces dans les conditions d'émergence d'une opinion publique dans la colonie. Nous discuterons également de la construction d'un climat de suspicion de la part des autorités entourant ces espaces. Nous verrons enfin que l'appareillage de surveillance étatique s'oriente d'abord sur la cooptation des tenanciers, maîtres de leur domaine situé à l'intersection du privé et du public.

\section{Émergence d'une culture de sociabilité semi-publique au Québec}

Les lettres au cher fils (1748-1753) d'Élisabeth Bégon ou bien la portion canadienne du journal (1749) du botaniste suédois Pehr Kalm constituent les sources principalement mobilisées pour se renseigner sur la vie mondaine de l'élite canadienne dans les dernières décennies du Régime français9 ${ }^{9}$ Ces textes ne font pas mention d'établissements commerciaux, comme les auberges, les cabarets ou même les cafés, que les élites administratives, militaires ou marchandes fréquenteraient pour leurs plaisirs mondains ou leur divertissement privé. Il existe bien dans les sources judiciaires un cafetier prénommé Pierre Hévé à Québec dans les années 1750, mais rien ne permet d'établir que son établissement était fréquenté par les élites ${ }^{10}$. En définitive, lorsqu’elle s'amuse en société, l'élite canadienne comme à Paris et dans les campagnes anglaises $^{11}$, le fait à son domicile ou bien chez l'un et chez

9. Élisabeth Bégon, Lettres au cher fils. Correspondance d'Élisabeth Bégon avec son gendre (1748-1753), Montréal, Hurtubise HMH, 1972; Jacques Rousseau, Guy Béthune et Pierre Morisset (éd.), Voyage de Pehr Kalm au Canada en 1749, Montréal, P. Tysseres, 1977.

10. Bibliothèque et Archives nationales du Québec, centre de Québec, fonds Nouvelle-France, Intendant, E1, S1, $\mathrm{P}_{22} 6$.

11. Roy Porter, Enlightenment, Britain and the Creation of the Modern World, Londres, Penguin, 2000, p. 266-267; Antoine Lilti, Le monde des salons. Sociabilité et mondanité à Paris au XVIII siècle, Paris, Fayard, 2005, p. 78-161; Angela Vanhaelen et Joseph P. Ward, Making Space Public in Early Modern Europe: Geography, Performance, Privacy, New York, Routledge, 2013, p. 41-59. 
l'autre $^{12}$. Que les administrateurs et les hauts gradés militaires soient accompagnés de leurs cuisiniers constitue le signe probant de la fréquence de ces pratiques. Ceux qui n'ont pas les moyens d'un tel luxe peuvent se replier sur un traiteur. À Québec et à Montréal, plusieurs individus exercent ce métier. Ces traiteurs proposent d'approvisionner les agapes de leurs clients directement chez les amphitryons ${ }^{13}$. C'est ce qu'on repère dans une petite annonce publiée en 1768 , en anglais et en français, dans La Gazette de Québec par un certain Alexandre Menut, ancien cuisinier personnel du gouverneur James Murray converti au métier de traiteur ${ }^{14}$.

Dans Life of Johnson, James Boswell rapporte ainsi les propos tenus par son ami, le lexicographe Samuel Johnson:

There is no private house, (said he) in which people can enjoy themselves so well, as at a capital tavern. [...] Whereas, at a tavern, there is a general freedom from anxiety. You are sure you are welcome: and the more noise you make, the more trouble you give, the more good things you call for, the welcomer you are. No servants will attend you with the alacrity which waiters do, who are incited by the prospect of an immediate regard in proportion as they please. No, Sir ; there is nothing which has yet been contrived by man, by which so much happiness is produced as by a good tavern or inn ${ }^{15}$.

Johnson différencie la sociabilité privée qu'il désigne comme caractéristique des pratiques françaises, et celle que fournit la tavern life, considérée comme le privilège des Anglais. Cette envolée participe à la construction du discours identitaire nationale britannique qui,

12. Thomas Anburey, Travels Through the Interior Parts of America; In a Series of Letters, Londres, William Lane, 1791, vol. I, p. 50-51. Anburey attend son édition critique. Néanmoins, il se dégage de son récit un regard sur une société coloniale nord-américaine qui reste très attachée aux codes sociaux inégalitaires européens; Arnaud Bessière, La domesticité dans la colonie laurentienne au XVII siècle et au début du XVIII siècle (1640-1710), thèse de doctorat, Montréal, Université du Québec à Montréal, p. 86-91; Catherine Ferland, «Le nectar et l'ambroisie: la consommation des boissons alcooliques chez l'élite de la Nouvelle-France au XVIII siècle », Revue d'histoire de l'Amérique française, 58, 4, 2005, p. 488-489.

13. Yvon Desloges «Jean Amiot, traiteur» et «Jacques Lemoine, traiteur» dans Gaston Deschênes et Denis Vaugeois, Vivre la Conquête à travers plus de 25 parcours individuels, Québec, Septentrion, 2013, t. 1, p. 7-14 et 78-86.

14. Gazette de Québec, 7 juillet 1768; John E. Hare, «Alexandre Menut», Dictionnaire biographique du Canada, en ligne, http://www.biographi.ca/fr/bio/ menut_alexandre_5F.html [page consultée le 24 janvier 2017].

15. James Boswell, Life of Johnson, Oxford, Oxford University Press, 2008, p. 697. 
comme l'a soutenu Linda Colley, est issu d'une série d'opposition à l'Autre : l'Écosse, la France, etc. ${ }^{16}$ En face de la France absolutiste, militariste et aux mœurs rigides, se tiendrait vaillamment une Angleterre parlementaire, commerçante et détendue. Il demeure qu'une commercialisation de la société s'opère dans les centres urbains britanniques, un phénomène qui ne s'exprime pas de manière aussi puissante à la même époque en France et dans ses colonies ${ }^{17}$. La détermination des pratiques commerciales existantes sous le régime français permet de souligner l'originalité des nouveaux espaces de divertissement introduits après la Conquête.

La distance entre les cultures commerciales s'observe au travers de ces témoignages portant sur le paiement. En 1785, le jeune marchand britannique Robert Hunter note sur un ton hautain que le prix fixé d'avance n'est pas encore une pratique répandue dans les auberges de campagne canadienne:

When you ask them [les aubergistes] what's to pay for dinner or supper (which consists always of milk and eggs - they have nothing else), "Ce qui vous plaira Monsieur" is sure to be their answer, and, of course, you are sure to pay them more than their things are worth ${ }^{18}$.

Au tournant du siècle, à Londres, les coffeehouses et eating houses fournissent la carte des prix à l'avance. Pierre de Sales Laterrière, à l'occasion d'un séjour londonien, prend le temps de décrire cette nouveauté qui, en 1810, n’est pas encore introduite ni au Bas-Canada ni en France: «[...] il y avoit près de là des cafés et des eating houses, qu'on appelle traiteurs à Paris; il y en avoit un à côté, qui avoit le nom d'honnête, où alloient tous les jours quantité de messieurs ${ }^{19}$. » La publication sur une carte imprimée des repas et des denrées offerts, y compris leur prix fixé d'avance, constitue une innovation que Laterrière prend le temps de noter: p. 6-7.

16. Linda Colley, Britons: Forging the Nation, 1707-1837, Londres, Pimlico, 1994,

17. Neil McKendrick, John Brewer et J.H. Plumb, The Birth of a Consumer Society: The Commercialization of Eighteenth-Century England, Bloomington, Indiana University Press, 1982, p. 9-33, 197-262 et 263-285.

18. Louis B. Wright and Marion Tinling, Quebec to Carolina in 1785-1786. Being the Travel Diary and Observations of Robert Hunter, Jr., a Young Merchant of London, San Marino, The Huntington Library, 1943, p. 25.

19. Bernard Andrès, Les mémoires de Pierre de Sales Laterrière suivi de Correspondances, Montréal, Les éditions Triptyque, 2003, p. 244-245. 
Le maître se fit un plaisir de m'expliquer toute chose, et me donna une carte des prix de chaque espèce d'aliment, par plat et demi-plat; de sorte qu'avant de demander une chose, je pouvois voir d'un coup-d'œil ce qu'elle coûteroit. À l'aide de cette carte, je pus calculer ma dépense et celle de mon fils: semaine commune, elle s'élèvera à 52 s. 6 d. sterling ${ }^{20}$.

Dans son célèbre Dictionnary of the English Language Samuel Johnson différencie les expressions « coffeehouse », « tavern » et «inn » de la manière suivante: 1) «Coffeehouse. n.f [coffee and house.] A house of entertainment where coffee is sold, and the guests are supplied with news-papers»; 2) «Tavern. n.s. [tavern, Fr. Taberna, Lat.] A house where wine is sold, and drinkers entertained»; 3) «Inn. n.f. A house of entertainment for travelers $»^{21}$. Si les distinctions sémantiques proposées par Johnson semblent minces, c'est bien parce que l'ambiguïté caractérise leur usage historique et pragmatique.

Pour Michael Eamon, suivant en cela Jürgen Habermas, l'accès à l'imprimé, sur le modèle londonien, constitue un critère distinguant le coffeehouse des autres espaces semi-publics similaires ${ }^{22}$. S'il est vrai que certains établissements se distinguent par une proximité entre leur tenancier et l'univers de l'imprimééc vient nuancer les raccourcis de perception. Par exemple, à Québec et à Montréal entre 1766 et 1825, on n'offre pas que du café dans les établissements s'affichant ou étant désignés comme coffeehouse, et des journaux sont aussi disponibles dans quelques tavernes plébéiennes au cours de la période ${ }^{24}$.

Ce n'est pas tant la nature des services offerts qui entraîne la distinction. Pour départager un type d'établissement d'un autre - qui en

20. Idem.

21. Samuel Johnson, A Dictionary of the English Language in Which the Words are Deduced from their Origin and Illustrated in their Different Significations by Examples from the Best Writers to Which are Prefixed a History of the Language and an English Grammar in Two Volumes, Londres, G. and J. Offor, 1785, vol. I.

22. Micheal Eamon, op. cit., p. 166.

23. Alexander Simpson tenancier du British Coffeehouse de Québec est abonné à La Gazette de Québec et fréquente régulièrement l'imprimerie de Gilmore et Brown. Bibliothèque et Archives Canada, Collection Neilson, Memorial kept by Brown \& Gilmore, $\mathrm{MG}_{24}$ B1 t. 49; Bibliothèque et Archives Canada, $\mathrm{RG}_{4} \mathrm{~B} 28$, Tavern licences, t. 59-69.

24. Bibliothèque et Archives Canada, Collection Neilson, MG24 B1 vol. 49, Memorial kept by Brown Æ Gilmore; Bibliothèque et Archives Canada, RG4 $\mathrm{B}_{2} 8$, Tavern licences, vol. 59-79. 
définitive se ressemblent tous -, ce sont la qualité de ces services et la perception sociale que la clientèle lui confère qui servent de critères. Le marchand Georges-Hyppolyte Le Comte Dupré écrit: "Nous avons samedy grand diner chez le bonhomme Pacheaux [Pachot] en l'honneur de l'anniversaire du buste de sa majeste [George III]. » Après le départ de la partie anglophone de l'assemblée, Dupré souligne: «[qu']ils ont abandonné le câfé pour travaillér avec plus de libertté a leur entreprize, contre les nouvelles loix » ${ }^{25}$. Lutilisation par Dupré de l'expression «café» peut être comprise comme une manière de poser à la respectabilité de l'agape. Pourtant, les certificats que Pachot obtient de ses concitoyens le désignent comme aubergiste et non comme cafetier ${ }^{26}$. Il n'est pas non plus abonné à La Gazette de Québec.

Les divertissements au quotidien et la sociabilité de l'élite diffèrent peu, en matière de contenu, de ceux exercés par ceux qui occupent le bas de l'échelle sociale. Boire, danser, jouer aux cartes, tout se ressemble si ce n'est que dans la forme et dans le décorr ${ }^{27}$. Le maintien de l'apparence de l'écart social revêt alors toute son importance. L'intervention d'un lecteur de La Gazette de Québec du 18 avril 1765 illustre bien cette ambivalence. George Ascato interpelle son lectorat, francophone et anglophone. Alors que «l'esprit de politesse et de société fournit à chacun mil moyens de faire un repas aussi splendide que divertissant», tout déraille au moment «[...] qu’animés par cet esprit de gaïeté que procure l'honête liberté d'un repas amical; [...] on s'excite, on se provoque par des santés et des rondes ${ }^{28} »$. On s'enyvre «d'une manière indécente que si l'on etoit en Cabaret [Tavern] ou en compagnie de gens les plus grossiers » et «on ne craint point, sur tout dans certain[e]s Auberge [Tavern], de se mettre en dépense, en cassant, brisant des meubles, souvent, précieux ».

Pour Samuel Johnson, ce qui caractérise la tavern life, c'est la garantie d'un service attentif et d'une atmosphère détendue que rend

25. Lettre de George-Hippolyte St-George Dupré à François Baby, 6 octobre 1774, Université de Montréal, division des archives, Poo58U11402.

26. Bibliothèque et Archives Canada, $\mathrm{RG}_{4} \mathrm{~B}_{2} 8$, vol. 59-61, licences à Louis Pachot, $1766-1769$.

27. Richard L. Bushman, The Refinement of America: Persons, Houses, Cities, New York, Vintage Books, 1992, p. 51-58.

28. La Gazette de Québec, 18 avril 1765. 
possible la nature commerciale du lieu ${ }^{29}$. Or, dans ce rapport marchand de proximité, dans cette société coloniale où l'apparence de distinction s'émousse, c'est autant la personnalité et les efforts déployés tant par le tenancier pour «entretenir» ses convives que la «qualité sociale» des convives qui forment la distinction. Le tenancier doit mettre tout autant d'attention dans la qualité de son service, puisque sans prix fixé d'avance, sa rémunération varie en fonction de la projection de respectabilité du client. Mais ces nouveaux espaces participentils à l'émergence d'une opinion publique au Québec?

\section{Émergences surveillées d'espaces publics suspects (1764-1783)}

L'historien David Zaret a proposé deux conditions permettant d'identifier l'émergence d'une sphère d'opinion publique. À la suite de l'historien du droit Michel Morin, transposons ce modèle à la Province de Québec et au Bas-Canada. Ces deux conditions sont les suivantes: 1) Présence d'une circulation libre d'imprimés; 2) Existence d'un dialogue public entre les idées véhiculées par ces imprimés et leurs consommateurs (lettrés ou non) $)^{30}$. Au Québec et au Bas-Canada, la première condition commence à être remplie en juin 1764. À ce moment, la première imprimerie à Québec met en circulation un premier hebdomadaire bilingue: The Quebec Gazette / La Gazette de Québec. À la suite de l'installation du gouverneur Guy Carleton à Québec en 1768, les imprimeurs s'astreignent à un régime d'autocensure. William Brown et Thomas Gilmore retirent tout débat sur les enjeux locaux de leurs gazettes publics. La pétition constitue le cas d'espèce. Le nouveau gouverneur entretient des vues bien arrêtées sur cette question. Il écrit à un de ses supérieurs : [...] je n’ai jamais encouragé cette tendance qu’ont les gens de pétitionner. » Carleton poursuit: «[...] le recours aux pétitions crée toujours l'impression qu'on arrache au gouvernement jusqu'aux mesures qu'il est juste et sage d'adopter sans qu'elles soient demandées. » Le danger ultime demeure que «[...]

29. James Boswell, Life of Johnson Unabridged, Oxford, Oxford University Press, 2008, p. 697, cité par Peter Thompson, Rum Punch and Revolution: Taverngoing and Public Life in Eighteenth-Century Philadelphia, Philadelphie, University of Philadelphia Press, 1999, p. 220 ; Bushman, op. cit., p. 160-164.

30. David Zaret, Origins of Democratic Culture: Printing, Petitions, and the Public Sphere in Early-Modern England, Princeton, Princeton University Press, 2000, p. 12-17. 
les gens s'habituent à former des associations et des coteries qui un jour ou l'autre peuvent se tourner contre le gouvernement ${ }^{31}$ ».

Le juriste Michel Morin a judicieusement relevé que les quelques pétitions qui circulent durant cette période sont élaborées par un petit nombre de personnes. Leurs signataires représentent un minime pourcentage de la population. Les pétitions elles-mêmes ne sont pas publiées avant 1788. Elles sont alors remises directement au gouverneur. Dans ces conditions, il n'existe pas de véritable sphère publique à l'intérieur de laquelle une opinion politique pourrait être diffusée, réfléchie, recyclée afin d'influencer les décisions de l'État ${ }^{32}$. Morin ne prend pas en compte les espaces physiques, les lieux de sociabilité où cette minorité peut émettre, à l'oral, en public, une opinion. Ceux qui fréquentent ces espaces, ces tavernes et ces cafés expérimentent certainement une facette de la sphère publique, celle de l'échange et de la rencontre.

Concernant l'univers populaire des alehouses, ces «cabarets à bière », de l'Angleterre du XVII siècle, Mark Hailwood relève que les discussions politiques participent de longue date de la culture de ces espaces. Cependant, la sphère publique qui s'opère dans ces lieux ne correspond pas à la formulation habermassienne classique, soit un espace caractérisé par une discussion ouverte, policée et libre dans laquelle une pluralité de vue peut s'exprimer. Clients et tenanciers sont prompts à dénoncer les propos séditieux. Le résultat, soutient Hailwood, est une culture politique qui n'est pas nécessairement antiautoritaire, hétérogène et fracturée ${ }^{33}$. Dans le contexte colonial québécois, cet interstice de parole suscite la suspicion d'une autorité nouvelle aux assises fragiles. Le pays conquis doit être maintenu

31. Lettre de Guy Carleton à Lord Hillsborough, 25 octobre 1769, Bibliothèque et Archives nationales du Canada, Q 6, p. 163-164, citée dans Michel Brunet, Les Canadiens après la Conquête, 1759-1775: De la Révolution canadienne à la Révolution américaine, Montréal, Fides, 1969, p. 204. Voir Gisela Giral, "Supplient très humblement... We Humbly Beg... » Les pétitions collectives et le développement de la sphère publique au Québec, 1764-1792, mémoire de maîtrise, Québec, Université Laval, 2013, p. $23-38$.

32. Michel Morin, «Les revendications des nouveaux sujets, francophones et catholiques, de la Province de Québec, 1764-1774", dans Essays in the History of Canadian Law. Volume XI: Quebec and the Canadas, Toronto, University of Toronto Press, 2013, p. 150.

33. Mark Hailwood, Studies in Early Modern Cultural Political and Social History. Volume 21: Alehouses and Good Fellowship in Early Modern England, Woodbridge, The Boydell Press, 2014, p. 69-72. 
sous son joug britannique face aux appétits de l'ancienne métropole française, menacé à partir de 1765 par les velléités révolutionnaires des bouillants voisins anglo-américains. Le gouverneur Guy Carleton impose, dès 1768, aux taverniers de la Province de Québec de prêter un serment d'allégeance à la couronne britannique ${ }^{34}$.

Carleton demeurait dubitatif quant à la loyauté des «nouveaux sujets» d'origine française lorsque viendrait le temps de mobiliser les forces à sa disposition en cas de crise ou d'invasion et n'estimait pas non plus les «anciens» sujets anglophones ${ }^{35}$. Les événements alimentent une atmosphère alarmiste. En avril 1775, le gouverneur a vent d'une réunion séditieuse au Montreal Coffeehouse tenu par James Crofton dans la Basse-Ville de Québec. Au cours de cette assemblée, le bouillant Thomas Walker, dont l'oreille fut tranchée en 1765 par un soldat qu'il était forcé de loger, aurait proféré des discours favorables au Congrès continental ${ }^{36}$. En septembre, les troupes congressistes marchent sur Montréal. Simon Sanguinet, royaliste montréalais, écrit dans son journal:

Lon vit la ville [Montréal] se remplir d'étrangers qui arrivoient tous les jours sous le titre de marchans - quoiqu'ils fussent réellement des officiers des Bastonnois qui avoient bloqué les retranchements à St. Jean qui étoient conséquemment autant d'espions ${ }^{37}$.

Alors que le lieutenant-gouverneur Hector Théophilius Cramahé est laissé responsable de la capitale avec une garnison restreinte, une proclamation est publiée dans cette ville afin, comme l'écrit Sanguinet,

34. Bibliothèque et Archives Canada, $\mathrm{RG}_{4}$ B28, vol. 59. Voir aussi l'endos des reconnaissances de Francis Babau, Pierre Dusau, Samuel Roupe, Nicolas Boulard, Charles Reschebach, Veuve de Barthelemy Hill et Donald Morrison, dans RG4 B28, vol. 6o-61, vol. 120 Shop licences; Bibliothèque et Archives nationales du Québec, centre de Québec, Fond Georges Allsopp, $\mathrm{P}_{313}, \mathrm{~S}_{2}$, Cahier de lettres envoyées par George Allsopp, $1768-1770$, p. 50, 52-53 et 81 .

35. Voir "Carleton à Shelburne, 20 janvier 1768 », dans Adam Shortt et Arthur G Doughty, Documents Relating to Constitutional History of Canada, Part. I, Ottawa, J. de L. Taché, 1918, p. 295; Brian Young, Patrician Families and the Making of Quebec: The Taschereaus and McCords, Montreal/Kingston, McGill/Queen's University Press, 2014, p. 63-66.

36. Bibliothèque et Archives Canada, $\mathrm{RG}_{4} \mathrm{~B}_{2} 8$. vol. 60 et 64 . Alfred Leroy Burt, The Old Province of Quebec, Toronto, Ryerson Press, 1933, p. 208-209.

37. Richard Ouellet et Jean-Pierre Therrien, L'invasion du Canada par les Bastonnois. Journal de M. Sanguinet, Québec, Ministère des Affaires culturelles, 1975 , p. 51-52. 
«d'examiner» ces gens. Cramahé «ordonne et commande rigoureusement à tous les hôteliers, cabaretiers, et toutes personnes qui recevront aucuns étrangers quelconques de donner les noms, le lieu des demeures, \&c. ${ }^{38}$ ». Les taverniers deviennent la cheville ouvrière du renseignement colonial dans l'enceinte de Québec.

En 1794, devant la crainte suscitée par l'agitation sociale, le gouvernement bas canadien entérine l'Alien Act sur le modèle d'une loi sanctionnée l'année précédente en métropole ${ }^{39}$. Cette loi oblige les étrangers se présentant sur le territoire à déclarer auprès des autorités leurs allées et venues. Dans les déclarations recueillies entre 1794 et 1811 s'égrènent les noms d'aubergistes et de taverniers. Par exemple, Pierre Antoine David, ancien soldat français stationné à Saint-Pierre et Miquelon, marin dans la marine britannique, déclare s'être retrouvé chez l'aubergiste Fournier de la Basse-Ville puis en pension chez le tavernier Joseph Bolvin des Trois-Rivières. Le parcours de Jean Lamort, marin originaire de Gonneville en Normandie, est éclairant. À l'été 1798 , sitôt débarqué du brick Seaforth à Beaumont, il prend résidence chez le tavernier Richard, un Français établit après la Conquête. Puis, on le retrouve chez un nommé Lacasse, pour ensuite retourner chez Richard, ensuite à Québec, chez Madame Magnès (ou Agnès?), aubergiste, pour terminer chez un certain Pascal ${ }^{40}$. Les autorités perçoivent les étrangers comme des vecteurs de contamination. Les points d'ancrage fixes, les tavernes, sont plus commodes à surveiller que les individus. C'est par ce moyen que l'État colonial entend mener ses mesures prophylactiques contre la contamination révolutionnaire.

\section{Surveiller la dissidence durant les années contre-révolutionnaires (1783-1825)}

Nancy Christie soutient qu'au cœur du projet contre-révolutionnaire colonial se trouve le renforcement du pouvoir patriarcal, tant symbolique que légal, sur l'espace politique, mais aussi domestique. L'objectif

38. Idem.

39. Murray F. Greenwood, Legacies of Fear: Law and Politics in Quebec in the Era of the French Revolution, Toronto, University of Toronto Press, 1993, p. 116-138; La Gazette de Québec, 5 juin 1794.

40. Déclarations de Pierre Antoine David et Jean Lamort, Bibliothèque et Archives Canada, Declaration Act, RG4, B45, bobine H-1154. 
sous-jacent à la politique d'anglicisation qui s’affirme après 1791 impliquerait un réalignement ferme vers l'autoritarisme ainsi qu'une réaffirmation accrue des formes hiérarchiques. La loi de 1802 sur les relations entre maîtres et serviteurs constituerait la pièce de résistance de ce projet patriarcal colonial. Christie souligne que sous le Régime français, les cas de servitude seraient limités à la domesticité et demeuraient considérés comme une matière d'ordre privé fondée sur une relation contractuelle. Sous le Régime britannique, toute relation de travail participe à la catégorie de servitude qui devient alors un principe central régissant l'ordre social. La loi de 1802 criminalise non seulement l'insubordination des serviteurs, apprentis, journaliers et autres employés occasionnels, rendant du coup les relations de travail plus coercitives, mais confère aussi une autorité accrue aux maîtres, aux maris et aux pères de famille. Enfin, Christie avance que ce système de gouvernance familiale (family governance) aurait eu une large influence dans le cadre colonial bas canadien, où, soutient-elle, «welldeveloped public spheres and strong institutional structures beyond the household were absent ${ }^{41}$.

Notre hypothèse est que l'étendue de ce projet patriarcal colonial englobe également les espaces semi-privés censés, d'après ce modèle de la family governance, présider à l'émergence d'une sphère publique. Nous posons que la personne du tavernier, en tant qu'acteur, maître d'un domaine privé qui se déploie également dans le domaine public, se voit chargée de multiples pouvoirs. Maître de sa femme, de ses enfants, de ses engagés et de ses esclaves, le tenancier revêt de plus les habits de surintendant de son petit forum privé et ceux d'auxiliaire gouvernemental dans l'observation des lois. Le tenancier doit tout voir, tout entendre et se garder des libelles et des déclarations séditieuses qui pourraient compromettre sa licence. Le renforcement du pouvoir patriarcal sur l'espace domestique par le biais d'une interprétation stricte du droit criminel britannique confère à l'expression master of the house une large portée légale. Ce pouvoir consolide le contrôle sur l'accès à un espace domestique, dont les limites se voient du coup définies. Quoique Christie soutienne qu'il ne soit pas aisé de saisir comment les hommes et les femmes ordinaires perçoivent le pouvoir culturel qu'implique l'expression langagière de «maître chez lui », il

41. Nancy Christie, art. cit., p. 342. 
demeure que la conviction partagée est qu'une personne doit se comporter de manière décente dans la maison d'un autre homme. Le maître doit entretenir un contrôle viril sur sa maison. Cet aspect ressort des récits de la violence ordinaire entendue dans les cours de justice du district de Montréal (Fig. 1).

Entre 1807 et 1825, le juge en chef du district de Montréal, James Reid, a entendu pas moins de 45 causes impliquant le témoignage d'un aubergiste, d'un tavernier ou d'un hôtelier, et ce, sur l'ensemble du territoire du district de Montréal dans le cadre des cours du banc du Roi. Si le corpus comprend quelques témoignages faisant référence à des aubergistes, ce n'est pas avant 1816 que les tenanciers eux-mêmes sont appelés à la barre des témoins. Avec une moyenne de 4,5 témoignages de tenancier sur une moyenne de moins d'une vingtaine de cas entendus par année par le juge Reid dans les deux cours, les tenanciers sont présents dans près du quart des affaires entendues, et ce, dans l'ensemble du district avec une concentration dans le Montréal intramuros. Il faut aussi noter que les agglomérations importantes de la Rive-Sud (Laprairie, Châteauguay, Blairfendie, Sabrevois, William Henry et Dunham) correspondent aux étapes des routes terrestres et fluviales le long du fleuve Saint-Laurent et de la rivière Richelieu qui mènent aux États-Unis.

Contester devant la justice le pouvoir qu'exerce un chef de famille dans les limites de son foyer devient une entreprise délicate ${ }^{42}$. Du côté des tavernes, ces maisons publiques (public houses), la démarche estelle différente? L'expression master of the tavern qui est aussi en usage revêt un sens tout aussi fort aux yeux de la justice ${ }^{43}$. L'espace semi-privé de l'auberge entretient une forme d'ambiguiité quant aux règles du «maître chez soi ». Lorsque l'aubergiste est présent, il est communément admis par les personnes présentes qu'il demeure maître de son domaine. Le 26 décembre 1824, Alexis Donai et son frère, Ambroise, entrent vers les 7 ou 8 heures du soir chez Godefroy Giroux, aubergiste à Blairfindie, dans le comté de l'Acadie. Giroux note qu’Alexis «était

42. Voir Bibliothèque et Archives Canada, James Reid Collection, MG 24, B 173, Criminal Cases, vol. 2 March 1817, bobine 8558. The King vs Jos. Vincelet; Prudent Vincelet\} On trial for Indictment for a Riot.

43. Bibliothèque et Archives Canada, James Reid Collection, MG 24, B 173, Criminal Cases, vol. 3 May 1820: Montreal, Oyer \& Terminer, p. 129-288, bobine 8559 , The King vs John Murry\} On trial of Indictment for Burglary $25 \mathrm{Dec}^{\mathrm{r}}$ in dwelling house of Andrew Allen. 
en train », alors que ce dernier lui demande à boire du rhum. Laubergiste exerçant son pouvoir de contrôle lui rétorque qu'il a déjà assez bu. Après quelques échanges avec un certain Lagacé qui le mettait au défi de se battre, Alexis Donai, de son propre chef, est « tombé bien fort sur des pieces [de mobiliers] qu'il y avoit dans dans la maison ${ }^{44} »$. Devant ce comportement perturbateur, Giroux, le maitre de la maison, prie Donai de rester tranquille ou de s'en aller.

Cependant, lorsque le maître s'absente, on assiste à une situation ambiguë. Le 9 novembre 1825, à Belœil, Catherine et Julie Beaudry, filles mineures d'Étienne Beaudry, aubergiste, en présence de Julie Beaudriare, se retrouvent toutes seules dans l'auberge. Étienne Beaudry, qui était sorti visiter le quatrième voisin, ordonne à ses filles « de ne pas ouvrir la barre durant son absence». «La barre » désigne ici une pièce ou dépense où sont conservées les réserves d'alcools, ainsi que souvent les recettes et objets de valeurs du commerce et non une barre verrouillant la porte ${ }^{45}$. L'auberge étant publique, tous peuvent y entrer. Malgré cet ordre, deux hommes qui ne sont pas connus des filles entrent dans l'auberge et demandent à se faire servir une chopine de rhum. D’après Julie Beaudriare, Catherine, l'ânée, aurait dit: «[...] qu'elle n'etoit pas capable de vendre». C'est ce qu'elle reprit un peu plus tard en affirmant qu'elle les servirait pour 15 sols. Alors que Jean-Baptiste Lemire voulait payer en monnaie les 15 sols, Jean-Baptiste Cartier profite de la situation et dit à son comparse : «allons lui passer notre belle piastre ». Sur cette entrefaite, Catherine Beaudry prend la pièce française et rend la monnaie «de 330 Sols un chelin \& 5 coppres». Bons joueurs,

44. Bibliothèque et Archives Canada, James Reid, MG 24, B 173: Criminal Cases, vol. 4 August-September 1824, bobine 8559, The King vs Basile Bechard; Abraham Remillard\} On Indictment for manslaughter in killing Alexis Donai.

45. Les dictionnaires du XVIII ${ }^{\mathrm{e}}$ siècle ne recensent pas les termes «barre» ou «barreau» comme comptoir de service fermé avec des barreaux. Le dictionnaire Littré (1872-1877) spécifie que le terme d'origine anglaise «Bar», soit un lieu public dans lequel on donne à boire, a pour étymologie: «Bar, au sens de comptoir, est le français barre, les comptoirs ayant des barres pour tenir éloigné le public». Quelques indices dans notre corpus attestent cet usage : «Il a été perd $\hat{u}[. .$.$] PORTE-FEUILLE$ [...] Qui nous l'aura trouvé et voudra bien l'apporter au barreau du Caffé de Simpson à la Basse-ville [...] » (La Gazette de Québec, 13 octobre 1766); "Que vers les 9 heures il s'est couché [près] du Poil [poêle] dans la chambre de la barre [...] en entrant dans la barre ou etoit pendu sa montre [...] », etc. (Bibliothèque et Archives Canada, James Reid MG 24, B 173, Criminal Cases, vol. 3 May 1821: Montreal, Oyer \& Terminer, The King vs Pierre Leclerc \& Mary Fraser\} On Indictment for Burglary, p. 598-600); Dictionnaire d'autrefois, ARTFL/CNRS/University of Chicago, en ligne. 
Cartier et Lemire laissent un pourboire de « 3 coppres ». Au retour du père, Catherine présente la pièce à son père qui l'examine et constate la contrefaçon : la pièce est en cuivre. La transcription du témoignage d'Étienne Beaudry offre les détails. Le père se lance à la poursuite des comparses, ce qui le mène de l'autre côté de la rivière Richelieu. Il y interpelle les deux hommes suspects en leur soumettant qu'ils ont été chez lui : «mais ils n'ont pas voulu le reconnoitre», ce sur quoi l'aubergiste les a fait arrêter ${ }^{46}$. Les deux prisonniers sont amenés devant le juge de paix, simultanément seigneur du lieu, Jean-Baptiste-René De Rouville ${ }^{47}$. Laccusation consiste en la circulation de monnaie contrefaite; seul Cartier est jugé coupable. L'insistance des témoins à préciser qu'Étienne Beaudry avait interdit d'ouvrir «la barre » en son absence, la forte réaction de l'aubergiste qui poursuit les deux comparses jusqu'à en traverser une large rivière comme la Richelieu pour aller les trouver jusque dans la maison d'un étranger; tout indique un souhait d'obtenir réparation, mais surtout un désir de venger la violation de son autorité patriarcale sur son foyer et sur ses filles mineures.

\section{Conclusion}

Pour conclure, nous avons proposé quelques éléments permettant d'établir qu'une culture de la taverne qui a d'abord émergé en Angleterre puis dans ses colonies nord-américaines a été transposée dans la Province de Québec à la Conquête. Cette culture est caractérisée par la commercialisation d'une offre de services spécialisés centrés sur les classes moyennes et les élites. Cette offre délimite un espace élitaire respectable différencié d'un espace populaire malfamé. La transposition de cette culture de la sociabilité publique élitaire vient se superposer à une culture de la sociabilité élitaire française déjà présente, axée d'abord sur la commensalité privée. C'est d'abord par le biais de la commercialisation de la sociabilité que s'opère cette installation. Cependant, ce processus rencontre plusieurs obstacles. Le contexte autoritaire qui s'établit dès l'installation du gouverneur Carleton en

46. Bibliothèque et Archives Canada, James Reid MG 24, B173, Criminal Cases, vol. 4, February-March 1825, bobine M-8560, The King vs J" B $^{\text {te }}$ Cartier ; J ${ }^{\text {n }}$ Be Lemire $^{\text {te }}$ On Indictment for uttering a counterfeit French Crown.

47. Serge Courville, "Jean-Baptiste-René Hertel de Rouville», Dictionnaire biographique du Canada, en ligne. 
1768 s'intensifie au début des guerres révolutionnaires à la fin du $\mathrm{XVIII}^{\mathrm{e}}$ siècle. Cette situation encourage l'articulation d'un discours de méfiance envers les espaces publics. Cette rhétorique s’applique aussi aux espaces semi-publics et vient consolider le système de régulation par licence et serment. Les aubergistes et les taverniers deviennent des surveillants privilégiés de leur forum privé. La justice fait régulièrement appel à eux comme témoins. Les tenanciers font aussi appel à la justice pour affermir leur autorité sur leur domaine. Cette privatisation du pouvoir de surveillance de l'État s'accompagne d'une diffusion du discours moral sur la consommation d'alcool, en particulier les spiritueux, dans les classes populaires. Il est pertinent de se demander si les objectifs déclarés d'un lourd et couteux appareillage administratif (licences, serment, reconnaissances, etc.) servant à encadrer ce vice atteignent leurs buts. Dans les faits, il est pertinent de se demander si ce système n'est pas d'abord mis en place pour servir de taxation directe plutôt que comme moyen efficace de régulation sociale.

FIGURE 1

Tableau des causes entendues par le juge James Reid dans lesquelles des taverniers ont comparu comme témoins dans le district de Montréal (1816-1825) ${ }^{48}$

\begin{tabular}{|c|c|c|c|c|c|c|c|}
\hline \multirow[b]{2}{*}{ ANNÉES } & \multicolumn{7}{|c|}{ Répartition par régions des causes entendues } \\
\hline & $\begin{array}{c}\text { Total de } \\
\text { causes }\end{array}$ & $\begin{array}{l}\text { Cité de } \\
\text { Montréal }\end{array}$ & Faubourgs & $\begin{array}{c}\text { Île de } \\
\text { Montréal } \\
\text { (Campagne) }\end{array}$ & $\begin{array}{l}\text { Rive- } \\
\text { Sud }\end{array}$ & $\begin{array}{l}\text { Rive- } \\
\text { Nord }\end{array}$ & $\begin{array}{l}\text { Trois- } \\
\text { Rivières }\end{array}$ \\
\hline 1816 & 1 & 1 & & & & & \\
\hline 1817 & 7 & 5 & 1 & & & 1 & \\
\hline 1818 & 7 & 1 & & 2 & 2 & 1 & 1 \\
\hline 1819 & 3 & & 1 & & 3 & & \\
\hline 1820 & 6 & 5 & 1 & & & & \\
\hline 1821 & 8 & 5 & & 1 & 2 & & \\
\hline 1822 & 2 & 1 & & & 1 & & \\
\hline 1823 & 1 & & & & 1 & & \\
\hline 1824 & 2 & & & & 2 & & \\
\hline 1825 & 8 & 5 & & & 3 & & \\
\hline Total & 45 & 23 & 3 & 3 & 13 & 2 & 1 \\
\hline
\end{tabular}

48. Bibliothèque et Archives Canada, James Reid Collection, MG 24, B 173, bobines 8558 à 8560 . 